Papers in Physics, vol. 14, ART. 140001 (2022)

Received: 26 March 2021, Accepted: 23 November 2021

Edited by: C. Brito

Reviewed by: A. Nicolas (ILM, CNRS \& Université Claude Bernard Lyon, France)

Licence: Creative Commons Attribution 4.0

DOI: https://doi.org/10.4279/PIP.140001

www.papersinphysics.org

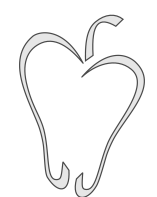

ISSN 1852-4249

\title{
Physical distance characterization using pedestrian dynamics simulation
}

\author{
D. R. Parisi ${ }^{1 *}$, G. A. Patterson ${ }^{1 \dagger}$, L. Pagni ${ }^{2}$, A. Osimani ${ }^{2}$, T. Bacigalupo ${ }^{2}$, J. \\ Godfrid $^{2}$, F. M. Bergagna ${ }^{2}$, M. Rodriguez Brizi², P. Momesso ${ }^{2}$, F. L. Gomez ${ }^{2}$, J. \\ Lozano $^{2}$, J. M. Baader², I. Ribas ${ }^{2}$, F. P. Astiz Meyer ${ }^{2}$, M. Di Luca², N. E. \\ Barrera $^{2}$, E. M. Keimel Álvarez ${ }^{2}$, M. M. Herran Oyhanarte ${ }^{2}$, P. R. Pingarilho ${ }^{2}$, X. \\ Zuberbuhler $^{2}$, F. Gorostiaga ${ }^{2}$
}

\begin{abstract}
In the present work we study how the number of simulated customers (occupancy) affects social distance in an ideal supermarket, considering realistic typical dimensions and processing times (product selection and checkout). From the simulated trajectories we measure social distance events of less than $2 \mathrm{~m}$, and their duration. Among other observables, we define a physical distance coefficient that informs how many events (of a given duration) each agent experiences.
\end{abstract}

\section{Introduction}

One of the measures widely applied to mitigate the Coronavirus disease (COVID-19) outbreak is social distancing; that is, maintaining a certain physical distance between people [1]. This distance acts as a physical barrier to droplets released from the nose or mouth of a potentially infected person. When another person is too close, they could breathe in the droplets and become infected. Although COVID-19 is our current concern, physical distancing could be useful for any contagious disease.

We should emphasize that a physical distance of 1-2 $\mathrm{m}$ is not sufficient for some other types of transmissionsuch as transmission by aerosols [2, 3] or fomites [3]. Moreover, many other important

\footnotetext{
*dparisi@itba.edu.ar

†gpatters@itba.edu.ar
}

1 Instituto Tecnológico de Buenos Aires (ITBA), CONICET, Lavardén 315 (1437), C.A. de Buenos Aires, Argentina.

2 Instituto Tecnológico de Buenos Aires (ITBA), Lavardén 315 (1437), C. A. de Buenos Aires, Argentina. factors, such as good ventilation (for indoor systems) and the use of face masks, are not included in our analysis.

Recent studies $[4,5]$ have suggested combining microscopic agent simulation with general diseasetransmission mechanisms. However, because of uncertainties and the complexity of current knowledge for quantifying COVID-19 transmission processes, here we will not consider any particular contagion mechanism. We will focus instead on studying the distance between people in an everyday pedestrian facility as an isolated aspect to be integrated in the future by experts considering all mechanisms for any particular disease propagation. Additionally, findings have been reported from recent physical distance studies that considered field data from a train station [6] and simulations of bottleneck scenarios [7].

One of the key questions we will try to answer is how to describe the physical distance for any given occupation of an establishment. To solve this problem, we must consider the displacements and trajectories of pedestrians while they perform certain tasks, thus the obvious tool to use is pedes- 
Papers in Physics, vol. 14, ART. 140001 (2022) / D. R. Parisi et al.

trian simulation. The time evolution of positions of simulated agents can provide not only the relative distance between agents, but also the duration of events in which the recommended social distance is not kept.

Many industries and shops have been closed in different phases of the COVID-19 pandemic. However, grocery shops have to be kept open, and supermarkets in particular. To prevent crowding and to keep some physical distance between customers, the authorities reduced the allowed capacity. Different countries' regulations have adopted social distance requirements between 1 and $2 \mathrm{~m}$ [6]. In the present study we will consider a distance of 2 $\mathrm{m}$ as the social distance threshold.

The main objective of this work is to introduce a methodology for characterizing and analyzing the physical distance between agents. We propose to investigate how the allowed capacity affects the physical distance between shoppers in an ideal supermarket of $448 \mathrm{~m}^{2}$. The results should not be extrapolated directly to other supermarkets or facilities; nevertheless, the methodology could be used with other trajectories based either on simulations or field data obtained from a pedestrian system.

\section{Models}

In order to simulate the complex environment and the agents' behavior, the proposed model involves three levels of complexity: operational, tactical, and strategic [8].

\section{i Strategic level}

The most general level of the model consists of a master plan for the agent when it is created. In practical terms, for the present system it gives a list of $n_{p}$ products for agents to acquire (a shopping list). Each of the $n_{p}$ items is chosen at random from a total of $m_{p}$ available products. Also, they are identified with a unique target location $\left(\mathbf{x}_{p n}\right)$ in the supermarket.

Once the agent is initialized with its shopping list, the strategic level shows the first item on the list to the agent. The agent will move toward it using the lower levels of the model. When the agent reaches the position of the product, it will spend a picking time $\left(t_{p}\right)$ choosing and picking up the product, after which the strategic level will present the next item on the list to the agent.

When the list of products is complete, the agent must proceed to the least busy supermarket checkout line. It will adopt queuing behavior until it gets to the checkout desk and spends time $t_{c o}$ processing its purchase.

\section{ii Tactical level}

The function of the tactical level is to present the agent with successive visible targets to guide it to the location of the desired product $\left(\mathbf{x}_{p n}\right)$ or checkout line. As input the tactical module takes the current agent position $\left(\mathbf{x}_{i}(t)\right)$ and the position of the current product $\left(\mathbf{x}_{p n}\right)$ on the list. The output is a temporal target $\left(\mathbf{x}_{v}(t)\right)$ visible from the current position of the agent. The definition of visibility is that if we take a virtual segment between $\left(\mathbf{x}_{i}(t)\right)$ and $\left(\mathbf{x}_{v}(t)\right)$, this segment does not intersect any of the walls or obstacles (shelves).

The information delivered by the tactical module is obtained by implementing a squared network connecting all the accessible areas of the simulated layout (see Fig. 2). For any pair of points within the walkable domain, the corresponding nearest points on the network are found and then the shortest path between these points is computed using the $\mathrm{A}^{*}$ algorithm [9].

Once the path in the network is defined, the temporary target $\mathbf{x}_{v}(t)$ is chosen as the farthest visible point on that path, seen from the current agent position. Clearly, $\mathbf{x}_{v}(t)$ will change with time, as the position of the agent changes. When the product target is visible from the agent's position, this is set as the visible target and the network path is no longer considered until a new product should be found.

\section{iii Operational level}

For the lowest level describing the agents' shortrange movements we propose an extended version of the Contractile Particle Model (CPM) [10]. This will provide efficient navigation to prevent potential collisions with other agents and obstacles. The basic model is a first-order model in which particles have continuous variable radii, positions and velocities that change according to certain rules. Specifically, the position is updated as 


$$
\mathbf{x}^{i}(t+\Delta t)=\mathbf{x}^{i}(t)+\mathbf{v}^{i} \Delta t
$$

where $\mathbf{v}^{i}$ is the desired velocity and $\mathbf{x}^{i}(t)$ the position at time $t$. The radius of the $i^{t h}$ particle $\left(r^{i}\right)$ is dynamically adjusted between $r_{\min }^{i}$ and $r_{\max }^{i}$. When this radius has large values, it represents the personal distance necessary for taking steps, but when it has low values it represents a hard incompressible nucleus that limits maximum densities.

When particles are not in contact, the desired velocity $\mathbf{v}^{i}$ points toward the visible target with a magnitude proportional to its radius,

$$
\mathbf{v}^{i}=\mathbf{e}_{t}^{i} v
$$

where the direction $\mathbf{e}_{t}^{i}$ and the magnitude $v$ are defined by the following equations:

$$
\begin{aligned}
\mathbf{e}_{t}^{i} & =\frac{\left(\mathbf{X}_{v}-\mathbf{X}^{i}\right)}{\left|\left(\mathbf{X}_{v}-\mathbf{X}^{i}\right)\right|}, \\
v & =v_{d}\left[\frac{\left(r-r_{\min }\right)}{\left(r_{\max }-r_{\min }\right)}\right],
\end{aligned}
$$

where $v_{d}$ is the desired speed.

While the radius has not reached the maximum $r_{\max }$, it increases at each time step, following

$$
\Delta r=\frac{r_{\max }}{\left(\frac{\tau}{\Delta t}\right)} .
$$

$\tau$ being a characteristic time at which the agent reaches its desired speed as if it was free, and $\Delta t$ is the simulation time step of Eq. (1). When two particles come into contact $\left(d_{i j}=\left|\mathbf{x}^{i}-\mathbf{x}^{j}\right|-\right.$ $\left.\left(r_{i}+r_{j}\right)<0\right)$ both radii collapse instantaneously to the minimum values, while an escape velocity moves the particles in directions that will separate the overlap:

$$
\mathbf{e}^{i j}=\frac{\left(\mathbf{x}^{i}-\mathbf{x}^{j}\right)}{\left|\mathbf{x}^{i}-\mathbf{x}^{j}\right|} .
$$

The escape velocity has the magnitude of the free speed and can thus be written as $\mathbf{v}_{e}^{i}=v_{d} \mathbf{e}^{i j}$. This velocity is only applied during one simulation step because, as the radii collapse simultaneously, the agents no longer overlap.
So far we have described the basic CPM as it appears in Ref. [10]. This model satisfactorily describes experimental data of specific flow rates and fundamental diagrams of pedestrian dynamics. However, particles do not anticipate any collisions, and this capacity is a fundamental requirement for simulating the ideal supermarket (displaying low and medium densities, and agents circulating in different directions). We therefore propose extending the calculation of agent velocity (Eq. (2)) by considering a simple avoidance mechanism.

The general idea is that the self-propelled particle will produce an action only by changing its desired velocity $\mathbf{v}_{i}(t)$, as stated in Ref. [11]. In this case, any change in the direction of desired velocity $\mathbf{v}$ through the new mechanism will depend on the neighbor particles and obstacles. First, the collision vector $\left(\mathbf{n}_{\mathbf{c}}{ }^{i}\right)$ is calculated as

$$
\begin{aligned}
\mathbf{n}_{\mathbf{c}}{ }^{i}= & \mathbf{e}^{i j} A_{p} e^{-d_{i j} / B_{p}} \cos \left(\theta_{j}\right) \\
& +\mathbf{e}^{i k} A_{w} e^{-d_{i j} / B_{w}} \cos \left(\theta_{k}\right)+\hat{\eta},
\end{aligned}
$$

where $j$ indicates the nearest visible neighbor, $k$ the nearest point of the nearest visible wall or obstacle, and $\hat{\eta}$ is a noise term for breaking possible symmetric situations.

Then the avoidance direction is obtained from

$$
\mathbf{e}_{a}^{i}=\frac{\left(\mathbf{n}_{\mathbf{c}}^{i}+\mathbf{e}_{t}^{i}\right)}{\left|\left(\mathbf{n}_{\mathbf{c}}^{i}+\mathbf{e}_{t}^{i}\right)\right|},
$$

and finally, the velocity of the particle to be used in Eq. (1), if particles are not in contact, is

$$
\mathbf{v}^{i}=v \mathbf{e}_{a}^{i}
$$

In Fig. 1 the vectors associated with the original and modified model can be seen in detail.

For the sake of comparison with force-based models, we also implement other operational models: the Social Force Model [12,13] and the Predictive Collision Avoidance (PCA) model [14]. The results for all three operational models are compared for selected observables, while the deeper study is performed using the rule-based model (CPM).

\section{a States of agents}

Because the agents must perform different tasks, more complex than just going from one point to 
A

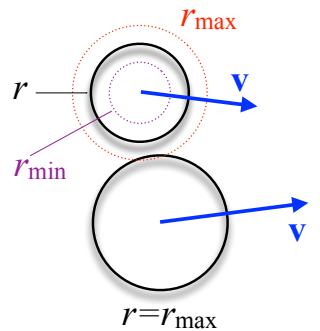

B

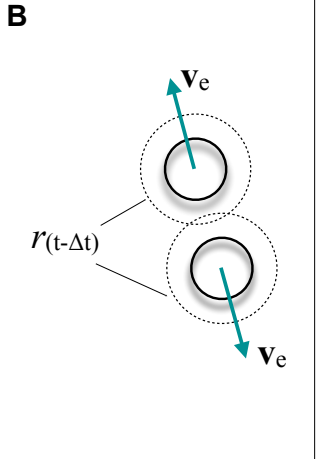

C

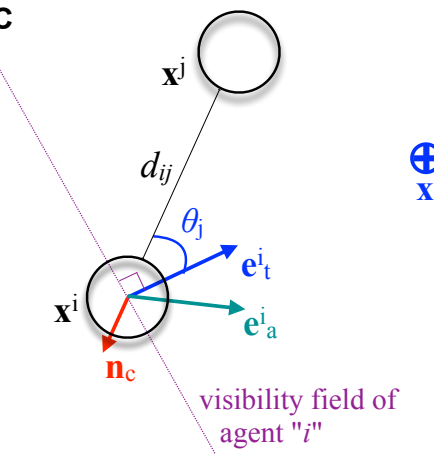

Figure 1: Contractile particle model. A: Two particles without contact. B: The radii of two particles that overlapped in the previous time step (dashed circles) collapse, and the particles take the escape velocity. A and $\mathrm{B}$ correspond to the original CPM. C: Modification considering an avoidance direction.

another, it was necessary to define five behavioral states. This was achieved by setting different model parameters and movement patterns. More concisely, the five behavioral states of agents were:

- Going: This is the normal walking behavior when going from one arbitrary point to another with the standard velocity and model parameters. Only in this state does the agent use the modified CPM velocity (Eq. (9)) to avoid potential collisions.

The other behavioral states use only the basic CPM (Eqs. (1) to (6)).

- Approaching: When the agent is closer than $2 \mathrm{~m}$ to the current product, it reduces its desired speed and, because of how parameters are set, it will not be forced to reach it if there is another agent buying a product in the same target $\mathbf{x}_{p n}$.

- Picking: Once the agent reaches the product (closer than $0.1 \mathrm{~m}$ ) a timer starts and it will remain in the same position (Eq. (1) does not update its position) until the picking time $\left(t_{p}\right)$ is up.

- Leaving: After spending time $\left(t_{p}\right)$, the agent leaves the current location and goes to the next product on the list. While abandoning this position it could find other waiting agents (in approaching behavioral state), so its parameters must be such that it can make its way through. Once the agent is farther than $2 \mathrm{~m}$ from the last product, it changes to the "going" behavioral state.

- Queuing: Finally, when the agent completes its shopping list it proceeds to the checkout desks by choosing the one with the shortest line. It waits at a distance of $1.5 \mathrm{~m}$ from the previous queuing agent, and when it reaches the checkout position it remains there for $t_{c o}$ time.

By considering these behavioral states in the agent model, the conflicts and deadlock situations are minimized. This model improvement thus enables us to simulate higher densities than with the basic operational models.

\section{Simulations}

The $448 \mathrm{~m}^{2}$ site of the ideal supermarket to be simulated is shown in Fig. 2. The dimensions of shelf $(1 \mathrm{~m} \mathrm{x} 10 \mathrm{~m})$ and aisle width $(2 \mathrm{~m})$ are taken
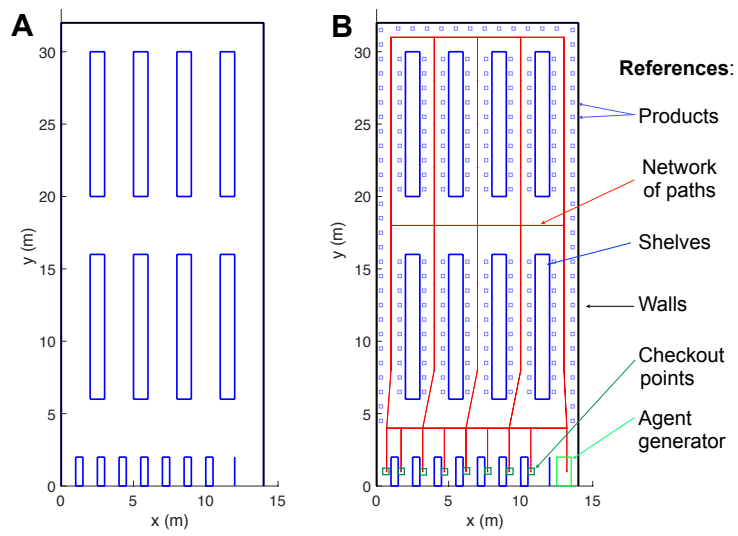

Figure 2: The ideal supermarket layout. A: Only walls and obstacles. B: The other model components as described in section II. 
Papers in Physics, vol. 14, ART. 140001 (2022) / D. R. Parisi et al.

Table 1: Parameters of the CPM operational model for all the behavioral states.

\begin{tabular}{lccccc}
\hline \hline Behavioral State & Going & Approaching & Picking & Leaving & Queuing \\
\hline$r_{\min }(\mathrm{m})$ & 0.1 & 0.1 & 0.2 & 0.1 & 0.1 \\
\hline$r_{\max }(\mathrm{m})$ & 0.37 & 0.35 & 0.2 & 0.3 & 0.12 \\
\hline$v_{d}(\mathrm{~m} / \mathrm{s})$ & 0.7 & 0.5 & 0 & 0.9 & 0 or 0.5 \\
\hline \hline
\end{tabular}

from typical real systems. The different processing times and other data considered were provided by an Argentine supermarket chain.

We define $N$ as the allowed capacity or the occupation of the supermarket; i.e., the total number of agents buying simultaneously inside the system. This is the most important input to be varied in our study and it ranges from $N=2$ to $N=92$. During the pandemic social groups are not allowed to enter commercial buildings, so we focus our study on single agents.

During the first wave of the pandemic there were long queues outside supermarkets, caused by capacity limitations, fear of shortages, and limited hours of operation. We therefore assume that outside the shop there is an infinite queue of clients who enter in order as the occupancy limit allows. The agent generator produces an inflow of 1 agent every $5 \mathrm{~s}$ until it reaches the $N$ value for the simulation. From that moment on, the agent generator monitors occupation, generating a new agent every time an existing agent completes its tasks and is removed from the simulation. By doing this, the value of $N$ is maintained constant over the entire simulation.

Every agent created by the generator is equipped with a shopping list of exactly $n_{p}=15$ items that, for simplicity, are chosen randomly from a total of 228 available items (shown in Fig. 2B). The corresponding product locations $\left(\mathbf{x}_{p n}\right)$ are separated by one meter from adjacent locations. Agents visiting the products on their lists spend a picking time with a uniform distribution $\left(\left(t_{p}\right) \in[60 \mathrm{~s}, 90 \mathrm{~s}]\right)$. After completing the lists, agents choose the shortest queue to one of the eight checkout points shown in Fig. 2B. The ideal supermarket has a maximum of four queues, each leading to two checkout desks. One of the strategies adopted in the supermarkets of Argentina was delimitation of the positions on the floor to guarantee the minimum physical distance $(1.5 \mathrm{~m})$ while queuing for checkouts. The first positions in these queues are at a distance of 3 $\mathrm{m}$ (at $y=4 \mathrm{~m}$, in Fig. 2) from the checkout points. Once an agent reaches the cashier (at $y=1 \mathrm{~m}$, in Fig. 2) it spends a checkout time $t_{c o}$ uniformly distributed between $t_{c o} \in[120 \mathrm{~s}, 240 \mathrm{~s}]$.

For each value of $N$ we simulated $2 \mathrm{~h}(7200 \mathrm{~s})$ and recorded the state of the system every $\Delta t 2=0.5$ s, thus producing 14400 data files with agents' positions, velocity, and behavioral state. The simulation time step $\Delta t$ used in Eq. (1) for all simulations was $\Delta t=0.05 \mathrm{~s}$. The noise term in Eq. (7) is a random vector, whose components $\eta_{x}$ and $\eta_{y}$ are uniformly distributed in the range $\eta_{x}=\eta_{y}=[-0.1 \mathrm{~m} / \mathrm{s}, 0.1 \mathrm{~m} / \mathrm{s}]$. And the relaxation time $\tau$ is set to $\tau=0.5 \mathrm{~s}$. The remaining model parameters depend on the behavioral state of the agent. For the case of "going", the parameters of the avoidance mechanism described in Eq. (7) are $A_{a}=1.25, B_{a}=1.25 \mathrm{~m}, A_{w}=15$ and $B_{w}=0.15 \mathrm{~m}$. The other behavioral states implement only the original CPM (without the avoidance mechanism) with the parameters displayed in Table 1.

\section{Results}

\section{i General aspects}

We first show general results of the simulated supermarket by displaying typical trajectories (Fig. 3) and density fields (Fig. 4). Figure 3 plots ten randomly chosen trajectories in the second hour of simulations for the selected $N$ values. Qualitatively, more intricate trajectory patterns can be seen as occupancy increases. However, in all cases it can be observed that the available area is uniformly visited by simulated agents while selecting the products on their list.

Complementary information is shown in Fig. 4, where density is averaged overl the entire simulation time $(2 \mathrm{~h})$. As expected, greater occupancy 
A

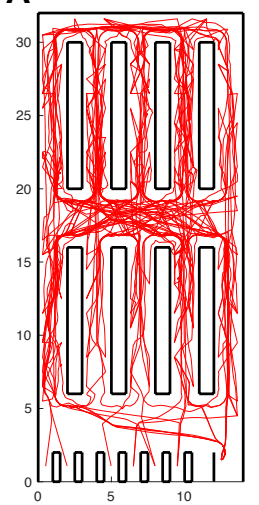

B

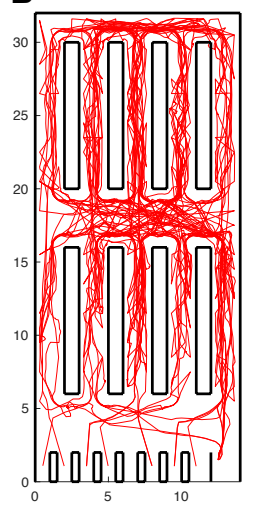

C

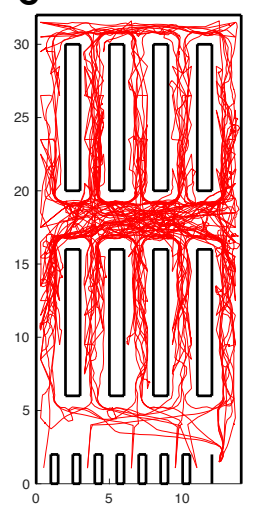

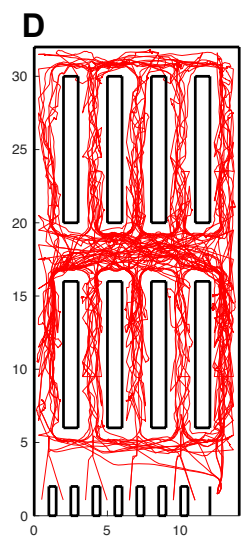

Figure 3: Ten random trajectories were chosen for different occupancies. A: $N=14, \mathrm{~B}: N=35, \mathrm{C}: N=62$, D: $N=92$.

presents higher mean density values. Moreover, these density fields present higher values at the spots where agents stay longer, thus revealing product selection points and predefined queuing places.

Also, as a macroscopic observable of the system, we study the number of agents that could be processed (i.e., complete the shopping list and exit the supermarket within the two hours simulated) and the mean residence time for those agents. These results are presented in Fig. 5. As can be observed, both quantities increase monotonically with the allowed occupancy for the studied range of values and the supermarket setup, considering eight checkout desks. Even though the agents purchase the same number of items, the trajectories generated present great variability in residence times.
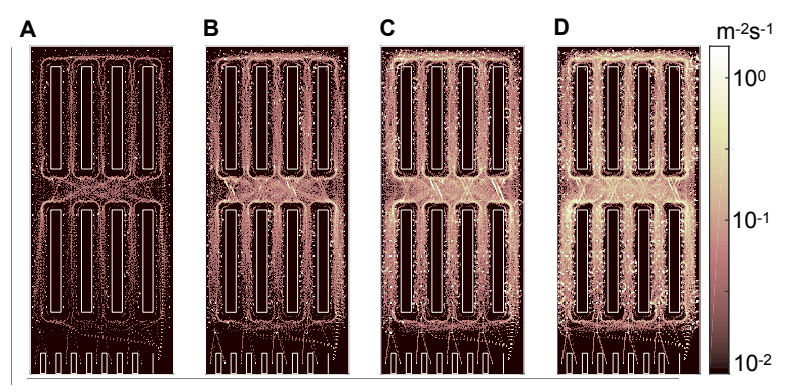

Figure 4: Density maps averaged over the $2 \mathrm{~h}$ simulation time for different occupancies. A: $N=14, \mathrm{~B}$ : $N=35, \mathrm{C}: N=62, \mathrm{D}: N=92$.
Furthermore, it can be seen that different operational models display similar observables. The SFM [12,13] and PCA [12,14] models are forcebased models that present more limitations in terms of the maximum density they can simulate before forces are balanced (generating deadlocks) for the complex scenarios and behavior considered. This is why the maximum occupancy studied with these models is lower than that simulated with the CPM described in section II.

\section{ii Distance analysis}

In this subsection we characterize the distance between agents during simulations with the modified CPM for different allowed capacities. An interesting outcome is the distance to the first neighbor for each agent shown in Fig. 6.

The probability density function (PDF) of firstneighbor distances $\left(d_{f n}\right)$ shows that for lower occupancy of the simulated supermarket, the probability of having the first neighbors further away than $d_{f n} \sim 5 \mathrm{~m}$ is greater. On the other hand, higher occupancy values generate higher probabilities of having a distance of less than $5 \mathrm{~m}$. In particular, all distributions show a maximum probable value around $d_{f n} \sim 4 \mathrm{~m}$. Moreover, the height of these probability peaks decreases for lower occupancy values.

Now we take the physical distance threshold of $2 \mathrm{~m}$, as discussed in section I, and calculate the related probabilities of agents below this critical 
Papers in Physics, vol. 14, ART. 140001 (2022) / D. R. Parisi et al.
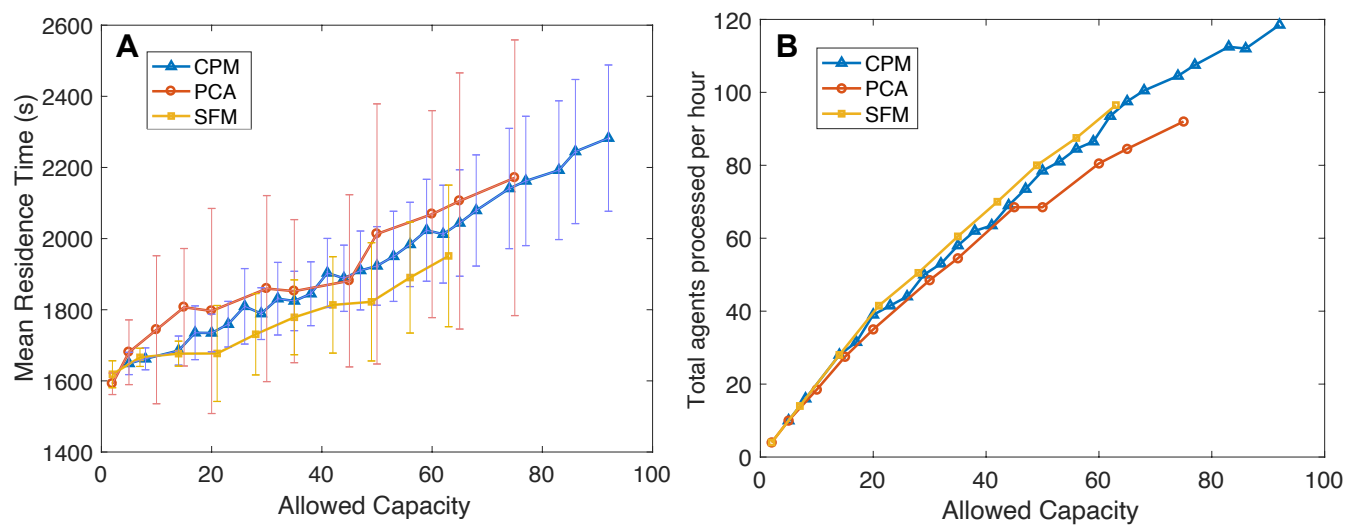

Figure 5: A: Mean residence time of agent as a function of occupation, for three different operational models. Error bars indicate one standard deviation. B: Number of agents processed per hour for the entire two-hour simulations, and also for the different operational models.

social distance. The first observable we calculate is the probability of the first neighbor being closer than $2 \mathrm{~m}\left(P_{f n<2 m}\right)$. In other words, this is the probability of having at least one neighboring agent within $2 \mathrm{~m}$. This is determined by averaging the data recorded every $\Delta t 2=0.5 \mathrm{~s}$, from minute 20 to 120 as shown in Eq. (10)

$$
P_{f n<2 m}=\frac{1}{n_{t i}} \sum_{t i=2400}^{t i=14400} \frac{n_{f n 2 m}}{N}
$$

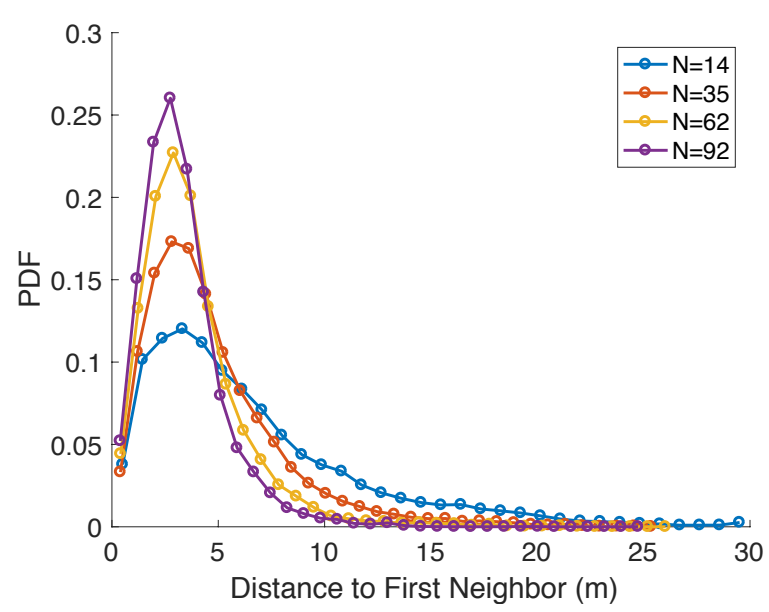

Figure 6: Probability density function of first neighbor distances. where $n_{t i}=12000=14400-2400$ is the data at recorded times after $20 \mathrm{~min}, N$ is the occupancy and $n_{f n 2 m}$ is the number of particles having a first neighbor at less than $2 \mathrm{~m}$. Note that if two particles $i$ and $j$ are the only particles at less than $2 \mathrm{~m}$, $n_{f n 2 m}=2$. Moreover, when $j$ is the first neighbor of $i, i$ will not necessarily be the first neighbor of $j$.

The above probability $\left(P_{f n<2 m}\right)$ only considers whether the first neighbor is closer than $2 \mathrm{~m}$; it does not consider whether there are many occurrences of neighbors at less than $2 \mathrm{~m}$. For this reason we now take into account the probability that a given pair of agents are within $2 \mathrm{~m}$ of one another $\left(P_{\text {pair }<2 m}\right)$

$$
P_{p a i r<2 m}=\frac{1}{n_{t i}} \sum_{t i=2400}^{t i=14400} \frac{n_{p 2 m}}{[N(N-1)] / 2}
$$

where $n_{p 2 m}$ is the number of pairs of particles at a distance closer than $2 \mathrm{~m}$ and $[N(N-1)] / 2$ is the total number of possible pairs having $N$ particles in the system. In this case, if only particles $i$ and $j$ are closer than $2 \mathrm{~m}, n_{p 2 m}=1$ because one pair is counted.

In Fig. 7 both probabilities $\left(P_{f n<2 m}\right.$ and $\left.P_{\text {pair }<2 m}\right)$ are displayed for the modified CPM and also for comparison with the SFM and the PCA model. It can be seen that the probability of having the nearest neighbor at less than $2 \mathrm{~m}$ increases monotonically with the allowed capacity. However, pair probability quickly increases for low oc- 

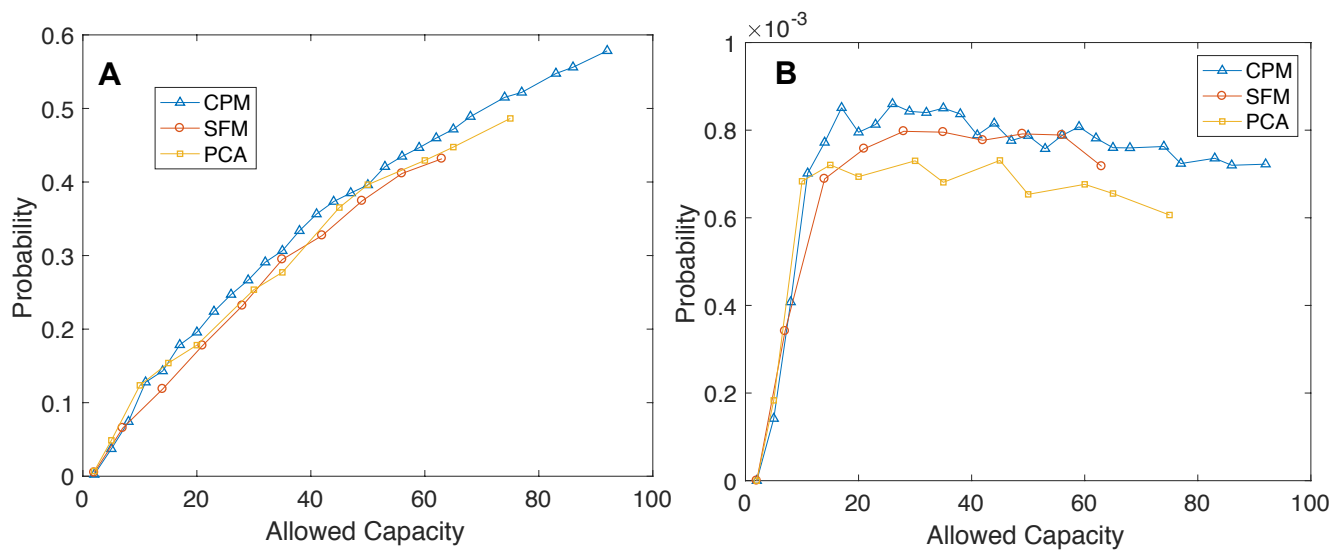

Figure 7: A: Probability of having the first neighbor closer than $2 \mathrm{~m}$ (Eq. (10)). B: Probability that a given pair of agents are within $2 \mathrm{~m}$ of one another (Eq. (11)).

cupancy, and after $N \sim 15$ remains almost constant, indicating that the number of pairs $n_{p 2 m}$ scaled with $N$ as the number of total possible pairs $\left(\sim N^{2}\right)$.

Furthermore, Fig. 7 indicates that different operational models display similar macroscopic behavior in terms of social distance, at least for values below or above $2 \mathrm{~m}$.

The above analysis focused on the occurrence of certain distances between simulated agents, but the duration of these events was not explicitly considered. This will be done in the following subsection.

\section{iii Duration of social distance events}

Here we study the time that events last when pairs of agents are found at less than $2 \mathrm{~m}$ (see section I). These events occur mainly when agents are selecting products at neighboring product locations or when queuing at the supermarket checkout. If two particles $i$ and $j$ meet at a given time and then separate by more than $2 \mathrm{~m}$, should the same particles meet up again at a future time this is considered two separate events.

Considering that: (a) The parameter we choose to maintain constant during each simulation is the allowed capacity $N$, and this capacity is reached at the beginning of each simulation in a very short time compared to other processes, and (b) all agents have the same number of items on their list, and thus the required time to complete it is similar on average, the first group of $N$ agents will go to the checkout points at nearly the same time, generating high checkout demand and long queues. Following this, the new agents will enter slowly as other agents exit the simulation, and thus the described behavior will relax. These dynamics lead to more queuing agents during the first hour of simulation and fewer during the second hour. We therefore analyze separately the duration of encounters occurring during the first and the second simulation hour in Fig. 8. The different time scales and the number of cases in both panels confirm that the first hour is dominated by particularly long queues waiting to check out, while in the second hour (Fig. 8B) social distance events of less than $2 \mathrm{~m}$ are dominated by the shorter process: product selection.

Events in the queuing line are long lasting for two reasons. First, the particular process at the checkout desk takes between 2 and 4 min (rather than the 1 to 1.5 min of the picking process). Second, a line with $n_{l}$ agents will make the last agents spend about $n_{l}$ times $t_{c o}$, which for a few agents, namely $n_{l}=5$, could represent 20 min waiting time at a distance of $1.5 \mathrm{~m}$ from another agent.

This problem of high exposure time between pairs of agents in queuing lines could be avoided if a slower rate of inflow of agents was adopted at the start of the process, let us say something above the maximum average outflow of the system (eight agents in three minutes, i.e., $\sim 1$ agent every $23 \mathrm{~s}$ ). We did not adopt this in the simulations because it would take too long for simulations to reach the 
Papers in Physics, vol. 14, ART. 140001 (2022) / D. R. Parisi et al.
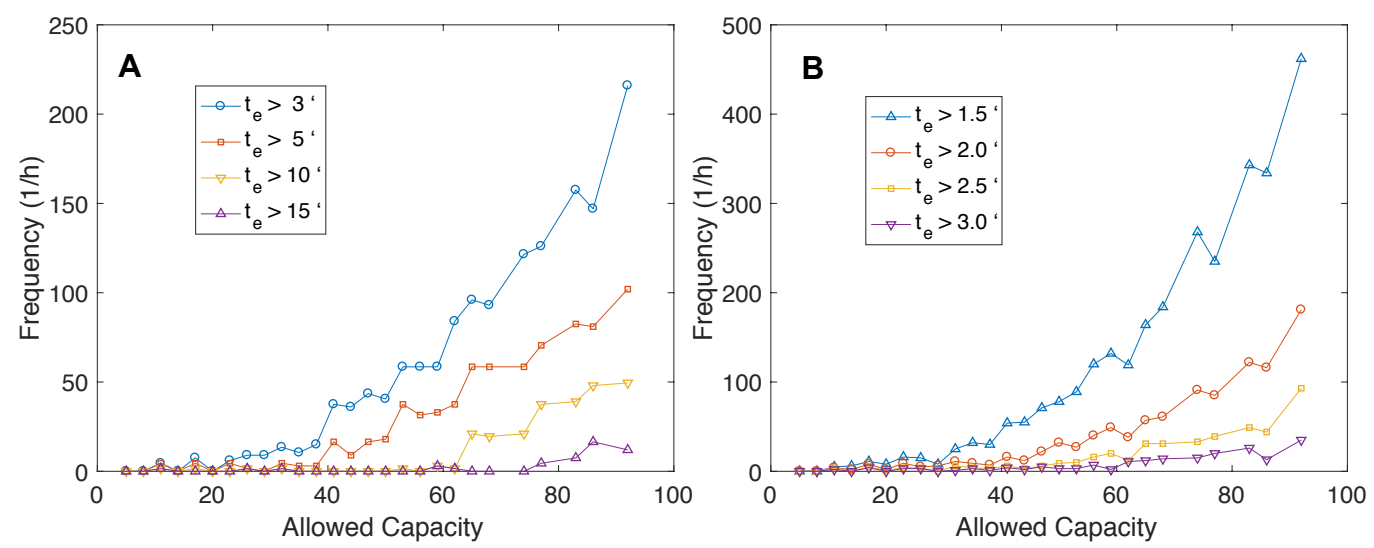

Figure 8: A: Number of events recorded in the first hour of simulations where two agents are at a distance of less than $2 \mathrm{~m}$ for more than $t_{e}$ min. B: The same measurement as $\mathrm{A}$ but for the second hour of the simulations.

desired occupation $N$. However, it is clear that the problem noted above at the beginning could be solved in a real operation by allowing a low flow rate of agents at opening time (of about twice the capacity of the checkout). Also, this transient behavior would represent a problem only at opening time, most of the daily operation being as described in our second simulation hour.

Furthermore, Fig. 8 shows that, as expected, fewer social distance events occur when the time thresholds increase. And in all cases, the number of events seems to grow quadratically with $N$.

\section{iv Physical distance coefficient}

Now, looking for a criterion that determines what a reasonable allowed capacity in the ideal supermarket would be, we define the physical distance coefficient $\left(\delta_{\pi}\left(t_{e}\right)\right)$ for the threshold distance of 2 $\mathrm{m}$, as

$$
\delta_{\pi}\left(t_{e}\right)=\frac{2 N_{e}\left(t_{e}\right)}{N_{p}}
$$

where $t_{e}$ is the minimum duration of a particular physical distance event $\left(r_{i j} \leq 2 \mathrm{~m}\right), N_{e}\left(t_{e}\right)$ is the number of these events that last at least $t_{e}$, and $N_{p}$ is the total number of agents processed by the system in the same period of time in which $N_{e}$ is computed. Factor 2 is needed to take into account the number of agents in the numerator, since two agents $(i$ and $j$ ) participate in each event.
This coefficient enables us to compare the number of agents who have participated in physical distance events of duration greater than $t_{e}$ with the number who have passed through the system. Thus a value of $\delta_{\pi}\left(t_{e}>2 \mathrm{~min}\right)=1$ indicates that, on average, each agent has participated in one event involving a physical distance of less than $2 \mathrm{~m}$ that lasts at least $2 \mathrm{~min}$. If $\delta_{\pi}\left(t_{e}>2 \mathrm{~min}\right)<1$, it would indicate that only a fraction of the agents have participated in such events.

Having established in section iii that the duration of events in the first simulation hour is dominated by the checkout line process, we now concentrate on looking at the second hour of simulation when the impact of these lines is very low and stationary. This situation is representative of the daily operation of the supermarket; this is shown in Fig. 9, which displays the physical distance coefficient as a function of occupation for different event duration limits $t_{e}$.

First, we note in Fig. 9A that the curve corresponding to $t_{e}>1$ min grows steeply with $\mathrm{N}$. This could be related to the fact that the picking time ranges between $1 \mathrm{~min}$ and $1.5 \mathrm{~min}$ and that the products are spaced by $1 \mathrm{~m}$, so if two agents aim simultaneously for the same product or the first or second nearest product, they could generate a 2 $\mathrm{m}$ physical event lasting at most $1.5 \mathrm{~min}$, and in particular many events lasting more than 1 minute would occur. Furthermore, the physical distance coefficient seems to follow a linear relation with $N$ 

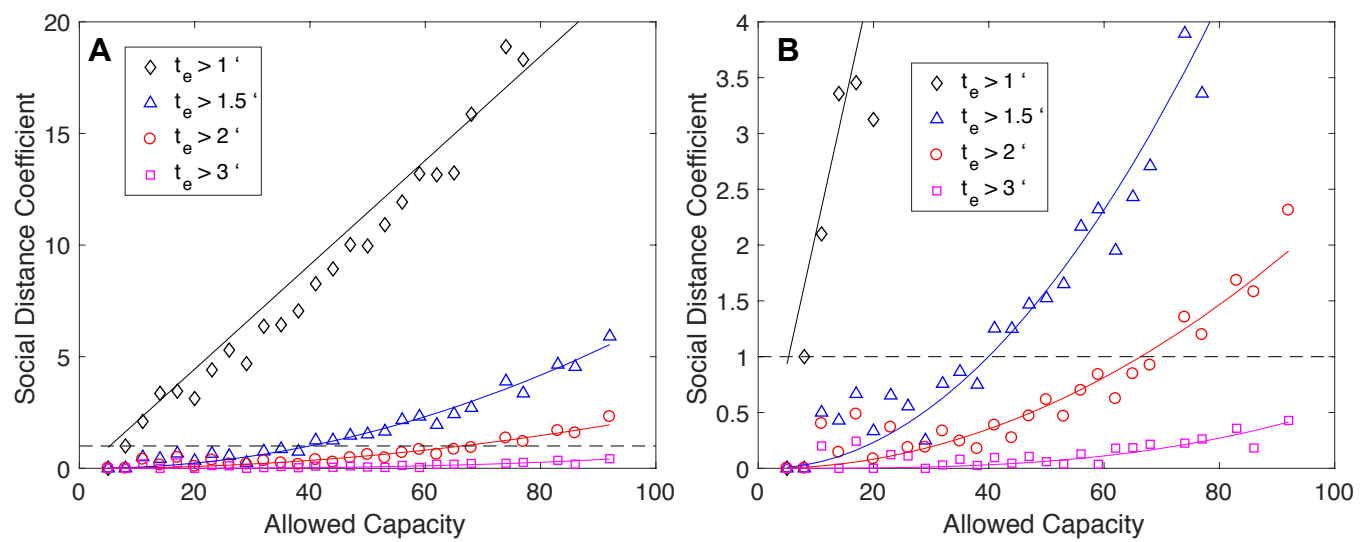

Figure 9: A: Physical distance coefficient as a function of supermarket occupation for the second simulation hour. B: Close up of previous figure showing details near $\delta_{\pi} \sim 1$. Solid lines correspond to the theoretical approach presented in section $\mathrm{V}$.

for this particular time limit $t_{e}$.

A change of regime can be observed for $t_{e}>1.5$ min, in which curves are more similar to one another for the different $t_{e}$ presented, and they follow a quadratic relation with $N$. Because the maximum picking time is $1.5 \mathrm{~min}$, this is the maximum possible overlapping time for two agents selecting neighboring (or the same) products. Longer lasting events will arise when more than two agents are waiting for neighbouring or the same products, as in the case of products near any of the short lines for checking out.

The results presented in Fig. 9B could be used as a guide for determining allowed occupancy. If based on epidemiological knowledge or criteria, it was determined that it would be acceptable for all agents to participate once in a $2-\mathrm{m}$ physical event lasting at most $1 \mathrm{~min}$, but then the allowed occupation would be very small, $N \sim 10$. Alternatively, if events up to $1.5 \mathrm{~min}$ were accepted, then the allowed occupation would be $N=40$. In the case of $t_{e}=2 \mathrm{~min}$, the capacity could rise to $N=70$. Also, it could be established that even for $N=90$ the events of the $2-\mathrm{m}$ physical distance, lasting more than 3 min, would affect only $40 \%$ of the processed agents.

Of course, Fig. 9B could be used to find another allowed occupancy if the criterion considered that, for example, only $25 \%$ of the agents could participate in the analyzed events.

\section{v Theoretical derivation of $\delta_{\pi}$}

In this subsection we theoretically derive the curves by interpolating the simulation data shown in Fig. 9. First we note that there are at least four sources of physical distance events, displaying increasing duration times:

- a very short time when two walking agents pass by in an aisle between shelves $\left(\sim 10^{0} \mathrm{~s}\right)$,

- a short time when conflicts appear due to lack of space $\left(\sim 10^{1} \mathrm{~s}\right)$,

- a longer time when agents are picking products at a neighboring or the same location $\left(\sim 10^{2}\right.$ s),

- a very long time when agents are queuing at neighboring positions in a (long) checkout line $\left(\sim 10^{3} \mathrm{~s}\right)$.

Because long lines can be avoided by suitable operation parameters, the analysis of $\delta_{\pi}$ in the above section was performed for the second simulated hour when checkout lines are kept to a minimum. Thus the longer process is related to agents selecting products at neighboring locations and will dominate the relationship between $\delta_{\pi}$ and occupancy.

The goal is to compute Eq. (12). We can write the numerator, $N_{e}\left(t_{e}\right)$, by taking into account the different time thresholds displayed in Fig. 9. 
Papers in Physics, vol. 14, ART. 140001 (2022) / D. R. Parisi et al.

First, we consider the case of events that emerge from the encounter of two agents during a time slot given by the mean picking time $\hat{t}_{p}=75 \mathrm{~s}$. We therefore calculate the average number of pairs of agents that go for the same product and are less than $2 \mathrm{~m}$ apart as

$$
N_{2}=\frac{733}{\left(\begin{array}{c}
2+m_{p}-1 \\
2
\end{array}\right)} \frac{N(N-1)}{2},
$$

where $m_{p}$ is the total number of available products, $\left(\begin{array}{c}2+m_{p}-1 \\ 2\end{array}\right)$ is the total number of possible ways of arranging two indistinguishable agents between the $m_{p}$ products, and 733 is the subset of these arrangements of two particles at less than $2 \mathrm{~m}$ away. The second factor corresponds to the total number of possible pairs for a given value of $N$.

Since the agents do not arrive simultaneously at their respective products, we compute the probability that the encounter of two agents lasts longer than $t_{e}$ as

$$
P_{2}\left(t_{e}\right)=\frac{\int_{t_{e}}^{\hat{t}_{p}} \mathrm{~d} T_{2}}{\int_{0}^{\hat{t}_{p}} \mathrm{~d} T_{2}}=1-\left(t_{e} / \hat{t}_{p}\right),
$$

where the denominator is the integral over the possible arrival times $T_{2}$ of the second agent, and the numerator is the integral over the possible arrival times that meet $\hat{t}_{p}>T_{2}>t_{e}$. Note that in this case the time $t_{e}$ will be limited to between $0<t_{e} \leq \hat{t}_{p}$; that is, on average the longest event is limited by the mean picking time $\hat{t}_{p}$. We then obtain the number of events $N_{e}\left(t_{e}>60 \mathrm{~s}\right)$, counting the number of time slots $\hat{t}_{p}$ within the observation time $T$, as

$$
N_{e}\left(t_{e}>60 \mathrm{~s}\right)=\kappa_{60} N_{2} P_{2}(60 \mathrm{~s}) T / \hat{t}_{p} .
$$

In our case $T=3600 \mathrm{~s}$ and $\kappa_{60}$ is a parameter that will be used to fit the model to the data, and could be interpreted as a correction considering that simultaneous events can occur during the same time slot $\hat{t}_{p}$, given that this discretization of time is just an approximation. Note that $\hat{t}_{p}$ is the average time that customers spend on the collection of products. If this time increases, customers will be immobile for a longer time. For this reason, increasing $\hat{t}_{p}$ decreases the number of encounters in a fixed period $T$.
Finally, the denominator of the $\delta_{\pi}$ is the number of processed agents $\left(N_{p}\right)$ in the same period of time $T$. Considering the picking time at each product, the number of products, the time needed to walk between them, and the waiting time at the checkout desk, a rough estimation of time needed for a free agent to complete its product list $\left(t_{r}\right)$ would be between 25 and $30 \mathrm{~min}$, as can be seen for low occupation in Fig. 5A. Thus, the number of processed agents per hour could be approximated as $N_{p} \sim T / t_{r} N \sim 2 N$. However, when occupancy increases, all internal processes become slower and as a consequence the effective proportionality constant between $N_{p}$ and $N$ decreases. Considering the result displayed in Fig. $5 \mathrm{~B}$, we approximate the proportionality constant by 1.5 and thus

$$
N_{p}=3 / 2 N
$$

Therefore, for events lasting more than $60 \mathrm{~s}$ we can write

$$
\begin{aligned}
\delta_{\pi}\left(t_{e}>60 \mathrm{~s}\right) & =\frac{2 N_{e}\left(t_{e}>60 \mathrm{~s}\right)}{N_{p}} \\
& =\kappa_{60} \frac{4 N_{2} P_{2}(60 \mathrm{~s}) T / \hat{t}_{p}}{3 N} \\
& \propto N
\end{aligned}
$$

Therefore, the functional dependence of $\delta_{\pi}(60 \mathrm{~s})$ on $N$ is linear, in accordance with the data shown in Fig. 9.

We then consider the case of events emerging from an encounter between three agents. Here, we calculate events that last longer than $\hat{t}_{p}$; this can only occur when three agents go together to the same product. The corresponding time slot for such events is $2 \hat{t}_{p}$. In this case, the average number of sets of three agents that go for products that are less than $2 \mathrm{~m}$ apart is

$$
N_{3}=\frac{m_{p}}{\left(\begin{array}{c}
3+m_{p}-1 \\
3
\end{array}\right)} \frac{N(N-1)(N-2)}{6},
$$

where the first factor comes from calculating the probability that three indistinguishable agents head towards the same product, and the second factor corresponds to the total number of sets of three agents. Only one pair of agents will have the chance 
to produce an event whose duration is longer than $\hat{t}_{p}$. This pair is made up of the two agents who arrived last, and the probability that the encounter of these agents lasts longer than $t_{e}$ is

$$
\begin{aligned}
P_{3}\left(t_{e}\right) & =\frac{\int_{t_{e}-\hat{t}_{p}}^{\hat{t}_{p}} \int_{t_{e}-\hat{t}_{p}}^{T_{2}} \mathrm{~d} T_{3} \mathrm{~d} T_{2}}{\int_{0}^{\hat{t}_{p}} \int_{0}^{T_{2}} \mathrm{~d} T_{3} \mathrm{~d} T_{2}} \\
& =\left(2-\left(t_{e} / \hat{t}_{p}\right)\right)^{2},
\end{aligned}
$$

with $\hat{t}_{p} \leq t_{e} \leq 2 \hat{t}_{p}$. Note that the arrival time of the second agent $T_{2}$ conditions the possible arrival time of the third $T_{3}$. Thus it is possible to calculate the number of events $N_{e}\left(t_{e}>90 \mathrm{~s}\right)$ and $N_{e}\left(t_{e}>\right.$ $120 \mathrm{~s})$ as

$$
\begin{aligned}
N_{e}\left(t_{e}>90 \mathrm{~s}\right) & =\kappa_{90} N_{3} P_{3}(90 \mathrm{~s}) T / 2 \hat{t}_{p}, \\
N_{e}\left(t_{e}>120 \mathrm{~s}\right) & =\kappa_{120} N_{3} P_{3}(120 \mathrm{~s}) T / 2 \hat{t}_{p} .
\end{aligned}
$$

In these cases the $\delta_{\pi}$ for events lasting longer than 90 and $120 \mathrm{~s}$ can be expressed as

$$
\begin{aligned}
\delta_{\pi}\left(t_{e}>90 \mathrm{~s}\right) & =2 \frac{N_{e}\left(t_{e}>90 \mathrm{~s}\right)}{N_{p}} \\
& =\kappa_{90} \frac{4 N_{3} P_{3}(90 \mathrm{~s}) T / 2 \hat{t}_{p}}{3 N} \\
& \propto N^{2}, \text { and } \\
\delta_{\pi}\left(t_{e}>120 \mathrm{~s}\right) & =2 \frac{N_{e}\left(t_{e}>120 \mathrm{~s}\right)}{N_{p}} \\
& =\kappa_{120} \frac{4 N_{3} P_{3}(120 \mathrm{~s}) T / 2 \hat{t}_{p}}{3 N} \\
& \propto N^{2} .
\end{aligned}
$$

Because sets of three particles are considered in Eq. (18), for $90 \mathrm{~s}$ and $120 \mathrm{~s} \delta_{\pi}$ grows with $N^{2}$, also according to the simulated data displayed in Fig. 9.

Finally, we repeat our analysis for the case of events originated by an encounter between four agents. We focus on events that last longer than $2 \hat{t}_{p}$; that is, events where the four agents go together to the same product. Again, the pair of agents who arrived last will have the chance to produce such an event. The average number of sets of four agents is

$$
N_{4}=\frac{m_{p}}{\left(\begin{array}{c}
4+m_{p}-1 \\
4
\end{array}\right)} \frac{N(N-1)(N-2)(N-3)}{24}
$$

and the probability that the encounter between the latest agents lasts longer than $t_{e}$ is

$$
\begin{aligned}
P_{4}\left(t_{e}\right) & =\frac{\int_{t_{e}-2 \hat{t}_{p}}^{\hat{t}_{p}} \int_{t_{e}-2 \hat{t}_{p}}^{T_{2}} \int_{t_{e}-2 \hat{t}_{p}}^{T_{3}} \mathrm{~d} T_{4} \mathrm{~d} T_{3} \mathrm{~d} T_{2}}{\int_{0}^{\hat{t}_{p}} \int_{0}^{T_{2}} \int_{0}^{T_{3}} \mathrm{~d} T_{4} \mathrm{~d} T_{3} \mathrm{~d} T_{2}} \\
& =\left(3-\left(t_{e} / \hat{t}_{p}\right)\right)^{3},
\end{aligned}
$$

with $2 \hat{t}_{p} \leq t_{e} \leq 3 \hat{t}_{p}$. The calculation for the number of events $N_{e}\left(t_{e}>180 \mathrm{~s}\right)$ is

$$
N_{e}\left(t_{e}>180 \mathrm{~s}\right)=\kappa_{180} N_{4} P_{4}(180 \mathrm{~s}) T / 3 \hat{t}_{p},
$$

and the $\delta_{\pi}$ for events lasting longer than $180 \mathrm{~s}$ is expressed as

$$
\begin{aligned}
\delta_{\pi}\left(t_{e}>180 \mathrm{~s}\right) & =2 \frac{N_{e}\left(t_{e}>180 \mathrm{~s}\right)}{N_{p}} \\
& =\kappa_{180} \frac{4 N_{4} P_{4}(180 \mathrm{~s}) T / 3 \hat{t}_{p}}{3 N} \\
& \propto N^{3} .
\end{aligned}
$$

Also, in this case the functionality dependence of $\delta_{\pi}(180 \mathrm{~s})$ seems to be in accordance with simulation results (Fig. 9). The scale laws for $\delta_{\pi}\left(t_{e}\right)$ are determined by the dominant encounter of agents; that is, the encounter that involves the lowest number of agents (which is the most probable event) and lasts longer than $t_{e}$. In fact, for the regime of $t_{e}>90$ $\mathrm{s}$ and $t_{e}>120 \mathrm{~s}$, we find the same scaling law, and this is because in these regimes the dominant encounter is that of three agents.

We calibrate these simulation data with Eqs. (17), (22), (23), and (27) by fitting the values of $\kappa$, and hence $\kappa_{60}=1.3, \kappa_{90}=1.7, \kappa_{120}=2.4$, $\kappa_{180}=1.5$. The solid lines shown in Fig. 9 stand for these results. The values obtained for $\kappa$ are reasonable in terms of interpretation of the fitting parameter proposed above, and indicate that our analysis is correct in terms of computing and the approximated value for the $\delta_{\pi}$ coefficient independently of the simulations, at least for the simple and idealized system studied. 
Papers in Physics, vol. 14, ART. 140001 (2022) / D. R. Parisi et al.

\section{Conclusions}

In this work we investigate and characterize social distancing in an everyday pedestrian system by simulating the dynamics of an ideal supermarket. Many sources of complexity were successfully taken into account with a multilevel model, which enables us to simulate not only translation but also more complex behaviors such as waiting times when selecting particular products and queuing at checkout points.

The main process that keeps pedestrians close to one another is the queuing lines for checkout. Therefore advice for the operation would be to keep these lines as short as possible either by increasing the number of checkout points or by decreasing occupancy.

At values greater than $2 \mathrm{~m}$, different operational models display similar macroscopic observables regarding social distance, indicating that the results are robust with respect to microscopic collision avoidance resolution, and also suggesting that the simulated paths of the particles are more influenced by the geometry, shopping list, and time-consuming process than by the particular avoidance mechanism. However, first-order models such as the CPM presented in Ref. [10] and section II.iii seem more suitable for simulation of highly populated scenarios with complex behavioral agents.

Taking a physical distance threshold of $2 \mathrm{~m}$, the probabilities and duration of such events are studied. The physical distance coefficient $\left(\delta_{\pi}\right)$ is defined as an indicator of the fraction of the population passing through the system that is involved in one or many of these events lasting at least a certain time threshold $t_{e}$. We put forward a theoretical analysis that satisfactorily fits the simulation data. It is important to note that applying this analysis requires an estimate of the number of agents processed per unit of time. In this work we use a relationship found from numerical simulations that can in the future be calibrated by empirical data or new models.

The same analysis can be carried out for a different set of parameters and for other pedestrian facilities such as other specific supermarkets or different systems (transport, entertainment, etc.). Of course, existing facilities can be monitored with measurement methods [6] providing high-quality trajectory data. This kind of data could also be interpreted in terms of the analysis performed in the present work.

The analysis presented takes into account only the duration of a given physical distance. As stated in the introduction, this is only a partial aspect of the contagion problem, and thus it must be integrated with other disciplines. For example, if a physical distance, a time threshold, and the fraction of the population that could be exposed to these conditions were determined, then maximum occupancy could be estimated using the observables defined in this work.

Acknowledgements - The authors acknowledge the information and data provided by the Argentinean Supermarket chain "La Anónima". This work was funded by project PID2015-003 (ANPCyT) Argentina and project ITBACyT-2018-42 and ITBACyT-2018-43 (ITBA) Argentina.

[1] World Health Organization, Coronavirus disease (COVID-19) advice for the public, Last updated: 4 June 2020.

[2] S L Miller, W W Nazaroff, J L Jimenez, A Boerstra, G Buonanno, S J Dancer, J Kurnitski, L C Marr, L Morawska, C Noakes, Transmission of SARS-CoV-2 by inhalation of respiratory aerosol in the Skagit Valley Chorale superspreading event, Indoor Air 31, 314 (2020).

[3] L Marr, S Miller, C Haas, W Bahnfleth, R Corsi, J Tang, H Herrmann, K Pollitt, and J L Jimenez, FAQs on Protecting Yourself from COVID-19 Aerosol Transmission, Last updated: 1 October 2020.

[4] K Rathinakumar, A Quaini, A microscopic approach to study the onset of a highly infectious disease spreading, Math. Biosci. 329, 108475 (2020).

[5] T Harweg, D Bachmann, F Weichert, Agentbased simulation of pedestrian dynamics for exposure time estimation in epidemic risk assessment, J. Public Health, 1 (2021).

[6] C A S Pouw, F Toschi, F van Schadewijk, A Corbetta, Monitoring physical distancing for crowd management: Real-time trajectory and group analysis, PLoS ONE 15, e0240963 (2020). 
Papers in Physics, vol. 14, ART. 140001 (2022) / D. R. Parisi et al.

[7] C M Mayr, G Köster, Social distancing with the optimal steps model, arXiv preprint, arXiv:2007.01634 (2020).

[8] S P Hoogendoorn, P H L Bovy, Pedestrian route-choice and activity scheduling theory and models, Transport. Res. B: Meth. 38, 169 (2004).

[9] J Yao, C Lin, X Xie, A J Wang, C C Hung, Path planning for virtual human motion using improved $A^{*}$ star algorithm, In: 2010 Seventh Int. Conf. on Information Technology: New generations, IEEE, Pag. 1154, Las Vegas (NV, USA) (2010).

[10] G Baglietto, D R Parisi, Continuous-space automaton model for pedestrian dynamics, Phys. Rev. E 83, 056117 (2011).
[11] R Martin, D Parisi, Pedestrian collision avoidance with a local dynamic goal, Collective Dynamics 5, 324 (2020).

[12] D Helbing, I Farkas, T Vicsek, Simulating dynamical features of escape panic, Nature 407 487 (2000).

[13] A Johansson, D Helbing, P K Shukla, Specification of the social force pedestrian model by evolutionary adjustment to video tracking data, Adv. Complex Syst. 10, 271 (2007).

[14] I Karamouzas, P Heil, P Van Beek, M H Overmars, A predictive collision avoidance model for pedestrian simulation, In: Motion in games, Eds. A Egges, R Geraerts, M Overmars, Pag. 41, Springer, The Netherlands (2009). 